Wilfrid Laurier University

Scholars Commons @ Laurier

Kinesiology and Physical Education Faculty

Publications

Kinesiology and Physical Education

$7-9-2020$

\title{
Sensory conflict alters visual perception of action capabilities during crossing of a closing gap in virtual reality
}

Natalie A. Snyder

Wilfrid Laurier University, natalieasnyder@gmail.com

Michael E. Cinelli

Wilfrid Laurier University, mcinelli@wlu.ca

Follow this and additional works at: https://scholars.wlu.ca/kppe_faculty

Part of the Motor Control Commons, and the Psychology of Movement Commons

\section{Recommended Citation}

Snyder N, Cinelli M. Sensory conflict alters visual perception of action capabilities during crossing of a closing gap in virtual reality. Quarterly Journal of Experimental Psychology. 2020;73(12):2309-2316. doi:10.1177/1747021820942212

This Article is brought to you for free and open access by the Kinesiology and Physical Education at Scholars Commons @ Laurier. It has been accepted for inclusion in Kinesiology and Physical Education Faculty Publications by an authorized administrator of Scholars Commons @ Laurier. For more information, please contact scholarscommons@wlu.ca. 


\section{Sensory conflict alters visual perception of action capabilities during crossing of a closing gap in virtual reality}

\begin{tabular}{|c|c|}
\hline Journal: & Quarterly Journal of Experimental Psychology \\
\hline Manuscript ID & QJE-STD-20-036.R2 \\
\hline Manuscript Type: & Standard Article \\
\hline $\begin{array}{r}\text { Date Submitted by the } \\
\text { Author: }\end{array}$ & 23-Jun-2020 \\
\hline Complete List of Authors: & $\begin{array}{l}\text { Snyder, Natalie; Wilfrid Laurier University, Kinesiology \& Phys. Ed } \\
\text { Cinelli, Michael; Wilfrid Laurier University, Kinesiology \& Phys. Ed }\end{array}$ \\
\hline Keywords: & $\begin{array}{l}\text { virtual reality, sensory conflict, collision avoidance, affordances, tau } \\
\text { coupling, aperture crossing }\end{array}$ \\
\hline
\end{tabular}

\section{SCHOLARONE" \\ Manuscripts}


RUNNING HEADER: SENSORY CONFLICT APERTURE CROSSING

Sensory conflict alters visual perception of action capabilities during crossing of a closing gap in virtual reality

Natalie Snydera, Michael Cinellia*

aDepartment of Kinesiology and Physical Education, Wilfrid Laurier University, Waterloo, ON, Canada

\section{Acknowledgement}

This project was funded through Natural Science and Engineering Research Council (NSERC)

Canada (05288-2014).

\section{Conflict of interest statement}

None.

Corresponding author at:

Michael Cinelli, Ph.D.

Associate Professor

Department of Kinesiology \& Physical Education

Wilfrid Laurier University

75 University Ave, W.

Waterloo, ON, Canada.

Tel.: +1 5198840710

fax: +1 5197474594 .

Email address: mcinelli@wlu.ca 
RUNNING HEADER: SENSORY CONFLICT APERTURE CROSSING

\begin{abstract}
The somatosensory, vestibular, and visual systems contribute to multisensory integration, which facilitates locomotion around obstacles in the environment. The joystick-controlled virtual reality (VR) locomotion interface does not preserve congruent sensory input like real-walking, yet is commonly used in human behaviour research. Our purpose was to determine if collision avoidance behaviours were affected during an aperture crossing task when somatosensory and vestibular input were incongruent, and only vision was accurate. Participants included 36 young adults who completed a closing-gap aperture crossing task in VR using real-walking and joystick-controlled locomotion. Participants successfully completed the task using both interfaces. Switch point between passable and impassable apertures was larger for joystick-controlled locomotion compared to real-walking, but time-to-contact (TTC) was lower for realwalking than joystick-controlled locomotion. Increased joystick-controlled locomotion switch point may be attributed to incongruency between visual and non-visual information, causing underestimation of distance travelled toward the aperture. Performance on future VR applications incorporating dynamically changing gaps can be considered successful using joystick-controlled locomotion, while taking into account a potential behaviour difference. Differences in TTC may be explained by the requirement of gait termination in real-walking but not joystick-controlled locomotion. Future VR studies would benefit from programming acceleration and deceleration into joystickcontrolled locomotion interfaces.
\end{abstract}

Key words: virtual reality; sensory conflict; collision avoidance; affordances; tau coupling; aperture crossing 
RUNNING HEADER: SENSORY CONFLICT APERTURE CROSSING

\section{Introduction}

During everyday locomotion, visual information is used to safely avoid obstacles (i.e., doorways) to reach a goal. One essential component of visual input is optic flow, which provides direct perceptual information about the rate and direction of movement of a person with respect to the environment as well as the objects in the visual field (Gibson, 2009; Lee \& Kalmus, 1980). When approaching a static object such as a doorway, optic flow allows individuals to directly perceive the time remaining before a collision will occur (i.e., time-to-contact). Time-to-contact (TTC) information aids the control of locomotion by using an individual's own perceptions of the properties of the doorway (size, shape, rate of movement, etc.), and of the individual themselves, to signal whether a change in locomotor behaviour is required to avoid a collision (Lee, 2012). In such situations, one must determine whether or not the gap created by the doorway is passable (i.e., its affordances). The possibilities for action that an object or situation offers are known as affordances, which could be based on the relationship between the dimensions of the observer and the dimensions of the object (Gibson, 1979). As a result, the dimensions of objects in the environment are specified based on body-scaled information (Fajen, 2013). For example, the ratio between aperture width and shoulder width determines the passability of the aperture (Warren \& Whang, 1987). Body-scaled information has been demonstrated to be useful for variables such as size and distance, specified as a ratio of eyeheight. As such, object height is specified as a multiple of the portion of the object that appears below eyeheight (Warren \& Whang, 1987). Standing eyeheight has a constant relationship with shoulder width, and therefore could determine the boundary between passable and impassable apertures. 
RUNNING HEADER: SENSORY CONFLICT APERTURE CROSSING

When the eyeheight of participants was raised without their knowledge, individuals perceptions of aperture passability reflected their apparent eyeheight (Warren \& Whang, 1987). Therefore, because action capabilities are based on the ratio between object size and body size, the perceptuomotor system must continuously update knowledge of these dimensions to adapt to the ever-changing environment (Hackney, Cinelli, \& Frank, 2014). This becomes more challenging with a changing ratio, such as when gaps are dynamically changing.

Past studies which investigated the visual control strategies that guide locomotion through dynamically changing gaps have used fixed-width, moving apertures (Cinelli, Patla, \& Allard, 2008), oscillating doors (Montagne, Buekers, Camachon, De Rugy, \& Laurent, 2003), and shrinking gaps (Fajen \& Matthis, 2011). Cinelli and colleagues (2008) and Montagne and colleagues (2003) found that when crossing oscillating doors, participants preferred to cross the aperture during the opening cycle, while decreasing their velocity to ensure safe and successful crossing. These behaviours do not fully reflect how individuals adjust their own action capabilities in a changing environment, as participants adjusted their crossing to optimize safety regardless of body size or locomotor capabilities. Thus, closing gap apertures allow for assessments of passability in a way that accounts for individuals' body size and locomotor capabilities. When perceiving the passability of closing gaps, visual information (optic flow) emerges from self-motion. When motion is self-produced, there is corresponding non-visual information (i.e., somatosensory and vestibular). This allows the actor to engage in action-scaling: that is, to interpret size, speed, distance, etc., in units of optic flow per step (Fath \& Fajen 2011). In fact, when visual gain (i.e., 
the speed at which subjects move through the environment) was manipulated such that participants experienced faster than normal self-motion, non-visual sensory information allowed for re-calibration of self-motion information to maintain accuracy of predictions based on a new rate of self-motion (Fajen \& Matthis, 2011). These results suggest that there are visual and non-visual contributions to perceptions of passability of closing gaps, and that manipulating sensory input can affect these perceptions.

The postural control system integrates visual and non-visual sensory information (i.e., somatosensory and vestibular) to ensure safe movement through the environment (i.e., multisensory integration; Chien, Eikema, Mukherjee, \& Stergiou, 2014). When perturbed, the postural control system is robust in its ability to recover from sudden loss of orientation information one or two systems (Assländer \& Peterka, 2016). Further, the contributions of each individual sensory system changes when available sensory information changes, and the recalibrations of these sensory contributions is referred to as sensory reweighting (Assländer \& Peterka, 2016; Karn \& Cinelli, 2018). Reweighting of sensory input is common in everyday life when closing one's eyes, or when travelling over a compliant surface (Assländer \& Peterka, 2016). Interestingly, when considering the unique contributions of each sensory system during locomotion, visual input is significantly upweighted during locomotion (Chien et al., 2014). This increased contribution of visual input reflects the importance of vision in the control of locomotion, and by extension, collision avoidance.

The objective of many past studies has been to perturb each sensory system to induce sensory reweighting, and determine how variables such as postural sway and sway variability are affected during locomotion. However, there remains an opportunity 
RUNNING HEADER: SENSORY CONFLICT APERTURE CROSSING

for further study of the functioning of the visual system when multisensory integration is perturbed or prevented, such as when perceiving passability of a closing aperture. Virtual reality (VR) platforms allow researchers to study the role of the visual system in performing a task when one or more non-visual sensory system is made incongruent (i.e., down-weighted), from the rest of the sensory systems, essentially decoupling multisensory integration. In virtual environments researchers are able to manipulate: the scene parameters; the interactivity of the individual within the environment; and/or the presented stimuli (van Veen, Distler, Braun, \& Bülthoff, 1998). In recent years, different locomotion techniques have been developed for VR systems to facilitate user-friendly navigation, which has subsequently expanded its usefulness for scientific research (Boletsis, 2017). One locomotion interface is real-walking locomotion, where the user interacts with the virtual environment inside a limited physical space while wearing the head-mounted display (HMD), which simulates the virtual environment. Using realwalking in VR preserves congruent and complete sensory information (Cirio, Olivier, Marchal, \& Pettré, 2013), which is advantageous, as it allows complete multisensory integration while performing tasks in the virtual environment. In VR environments where small physical space limits use, other interfaces have been developed which do not preserve all sensory input involved in locomotion and aperture crossing, but make up for the limited space available in VR designs. Such locomotion interfaces use a handheld joystick to simulate movement, which introduces incongruent sensory input from the vestibular system and somatosensory system (as the participant stands stationary in the laboratory environment, but visually perceives movement), but gain greater movement within the virtual environment (Slater, Usoh, \& Steed, 1995). 
As virtual locomotion relies predominantly on visual feedback, the elements of vision and perception of the virtual environment (i.e., field of view, optic flow) largely dictate behaviour outcomes (Cirio et al., 2013). The knowledge that visual information is heavily prioritized during virtual locomotion led to the purpose of this study, which was to evaluate young adults' behaviours on a closing-gap aperture-crossing task, when visual and non-visual information were incongruent (i.e., joystick-controlled locomotion) compared to when all sensory systems were congruent (i.e., real-walking, preserving optimal multisensory integration). We hypothesized that participants would be able to complete the task similarly using primarily visual information regardless of whether visual and non-visual information are congruent or not, reflected by similar aperture crossing behaviours and time required to make decisions.

\section{Materials and Methods}

\section{Participants}

Neurotypical young adults $(\mathrm{N}=36)$ were recruited to participate in the study (Table 1). Participants were included if they had normal or corrected-to-normal vision and were free from any injuries that would prevent them from physically walking through the simulated environment, and/or controlling their movement using a joystick. This study was approved by the Wilfrid Laurier University Research Ethics Board (REB \#5764).

\section{Protocol}

Written informed consent was obtained from all participants prior to start of the study in compliance with the university's Research Ethics Board. Participants were outfitted with an HTC Vive ${ }^{T M}$ VR system HMD (tethered HDMI cable allowing free 
RUNNING HEADER: SENSORY CONFLICT APERTURE CROSSING

movement up to $10 \mathrm{~m}$, not wireless) in order to provide an immersive VR environment experience. The HMD resolution was 1080 x 1200 pixels per eye, refresh rate $90 \mathrm{~Hz}$, field of view 110 degrees. Smooth motion was used (i.e., no additional visual motion) to ensure the scene was visually similar between the two locomotion interfaces. Once the HMD was placed on the participants' heads, they were allowed to walk around and free explore the VR environment to allow them to become comfortable and familiar within the simulated environment. Following the familiarization period and prior to the start of the experimental trials, participants completed 5 unobstructed steady-state walking trials of $7 \mathrm{~m}$ with no changes to the VR environment. The steady-state walking trials were needed to establish each participant's normal walking speed. Next, the participants completed 84 experimental trials: two blocks of 21 randomized walking trials and two blocks of 21 randomized trials using joystick to simulate locomotion through the VR environment. The four trial blocks alternated between locomotion type (real-walking or joystick-controlled) and starting locomotion type was counter-balanced between participants. For example, participant number 1 would perform one block of 21 realwalking locomotion trials, then one block of 21 joystick-controlled locomotion trials, and repeat this order for a total of four blocks (84 trials). Then, participant number 2 would perform one block of 21 joystick-controlled locomotion trials, then complete one block of 21 real-walking locomotion trials, and repeat this order for a total of four blocks (84 trials). Having trials organized in blocks that alternated between locomotion interfaces washed out any potential trial effects by strategically break up trials of each locomotion interface, and avoided oversaturation of either locomotion type. If not alternated, participants would have completed all 42 trials of each interface in a row. Participants 
RUNNING HEADER: SENSORY CONFLICT APERTURE CROSSING

were instructed to maintain their normal steady-state walking speed throughout all the walking trials and to avoid altering this speed for any reason. Additionally, each participant's normal steady-state walking speed was set as the constant movement speed during the joystick walking condition. Participants were given breaks when needed. Position data of the participants' head in space was recorded throughout the trial by the HTC Vive ${ }^{T M} \mathrm{HMD}$ at $90 \mathrm{~Hz}$ in order to determine the participants' behaviours.

\subsubsection{Experimental Design}

An aperture crossing task was selected for the experimental trials. The VR environment simulating the aperture crossing task was designed using Unity software. The VR environment simulated the interior of an industrial building with set of large factory doors (4 m aperture width, $4.3 \mathrm{~m}$ tall; Figure 1) located $5 \mathrm{~m}$ from the participants' starting location and a goal (i.e., square on ground) located $1 \mathrm{~m}$ beyond the doors. Participants were instructed to walk (at their comfortable steady-state speed) towards the goal and not get hit by the doors. Participants were requested to maintain their comfortable and consistent walking speed throughout each trial and avoid speeding up or slowing down as much as possible. During the approach, the doors would begin to close as soon as the participants travelled $1 \mathrm{~m}$ (i.e., $4 \mathrm{~m}$ from doors) regardless of locomotion type. The closing rate of the doors on any trial was set at one of seven different speeds, determined by a multiplication factor based on each participant's steady-state walking speed (i.e., closing rate=walking speed *1.2, 1.1, 1.0, 0.9, 0.8, 0.7, or 0.6 ). For example, if a participant approached the goal at $1 \mathrm{~m} / \mathrm{s}$ and the closing rate of the doors was $1.2^{*}$ walking-speed, the doors would close at $1.2 \mathrm{~m} / \mathrm{s}$ and therefore be closed prior to the time when the participant would reach them. The closing rates of the 
RUNNING HEADER: SENSORY CONFLICT APERTURE CROSSING

doors were chosen ensured that at least one condition would be impossible to pass through (i.e., $1.2^{*}$ ), and one should be passable $100 \%$ of the time (i.e., $0.6^{*}$ ). If participants decided the doors were passable without requiring a body rotation or increase their walking speed, they were instructed to pass through the aperture created by the doors and continue to the goal. If they decided the doors were impassable, they were instructed to stop their approach as soon as the decision was made. Participants completed the task using real-walking, where they physically moved through the VR environment, and also using joystick locomotion, where they would press a trigger on a handheld joystick to initiate movement (held at a constant velocity equal to the participant's comfortable walking speed), and would terminate movement by releasing the trigger. No physical locomotion was performed by the participant during joystickcontrolled locomotion. Instead, they stood stationary at the starting location and used the joystick to locomote.

\subsubsection{Data and Statistical Analysis}

For each locomotion interface, the proportion of trials (out of 6 per condition) in which each participant passed through the doors (0) or stopped (1) was recorded. Participant location at a change in speed (i.e., a stop) as well as which decision was made (pass through or stop) was determined by the HMD head position. Participant location when a stop occurred was recorded by the HMD as the distance between the participant and the aperture or recorded as a 0 if the participant passed through. No researcher feedback regarding aperture passage success was communicated to the participant. Within each interface, the proportion of trials in which a stop occurred within each of the 7 conditions (i.e., door closing speeds) was plotted, and a third order 
polynomial was generated. This was to determine the $50 \%$ switch point where a change in behaviour occurred (by solving for $\mathrm{x}$ where $\mathrm{y}=0.5$ ), or the aperture condition where participants decided to pass through and stop an equal amount of times.

The average time to contact (TTC), or the time remaining before the participant would have reached the doors, was calculated for the door closing rates of $1.0^{*}, 1.1^{*}$, and $1.2^{*}$ (walking speed) because participants stopped their approach during all trials, which allowed for analysis of TTC. This analysis was not possible for aperture conditions where a stop occurred less than $100 \%$ of the time (i.e., intermediate aperture closing speeds such as $0.8 / 0.9^{*}$ walking-speed), as a pass through was recorded as a 0 for head position. TTC (s) was calculated using each participants' head position relative to the doors when they completed a stop $(\mathrm{m})$ divided by their steady-state walking speed $(\mathrm{m} / \mathrm{s})$. For each participant, a $50 \%$ switch point and TTC values for each of the three aperture conditions were calculated for real-walking and joystick-controlled locomotion. Joystick and real-walking were compared using a Wilcoxon signed-rank test to determine any overall differences in the $50 \%$ switch point. To compare TTC values for the door closing conditions of $1.0^{*}, 1.1^{*}$, and $1.2^{*}$ (walking speed) between the two locomotion interfaces, a $2 \times 3$ repeated measures ANOVA was performed. To determine if there was a block order effect on behaviors, two one-way repeated measures ANOVAs were performed to compare the $50 \%$ switch point and the three TTC values across the four blocks of trials. Results were reported as mean \pm standard deviation; $p$ values $<.05$ were accepted as significant; Cohen's $d$ (t-tests) and Cohen's $f$ (rmANOVA) were reported for effect size. 
RUNNING HEADER: SENSORY CONFLICT APERTURE CROSSING

\subsection{Results}

Throughout all trials in this study, there were no virtual collisions with the doors, and no participants reported motion sickness. The approach speed of each participant throughout the duration of each trial was not recorded, but was monitored by researcher observation. Therefore, this study lacks the ability to confirm that participants walking speed was unchanged throughout each trial. However, the increments of closing speed of the doors were in $10 \%$ intervals of each participants average approach speed. Typically, people do not vary their approach speed from trial-to-trial by a greater amount than $10 \%$. Participants' comfortable walking speed in VR was calculated from steadystate walking trials, and ranged from 1.10 to $1.75 \mathrm{~m} / \mathrm{s}(\bar{x}=1.4 \pm 0.23 \mathrm{~m} / \mathrm{s})$, equal to previously recorded average human walking speeds during walking in a real environment (Montufar, Arango, Porter, \& Nakagawa, 2008).

The data for $50 \%$ switch point for real-walking and joystick-controlled locomotion failed a normality test. Therefore, the Wilcoxon signed-rank test revealed a significant difference in $50 \%$ Switch Point overall between walking and joystick $\left(t_{(34)}=4.4, p<.001\right.$, $d=0.52)$, such that the $50 \%$ Switch Point for walking $(\bar{x}=0.80 \pm 0.08)$ was significantly lower than for joystick $(\bar{x}=0.86 \pm 0.06$; Figure 2$)$.

The TTC results revealed a main effect of condition $\left(F_{(2,70)}=5.517, p=.006\right.$, $f=0.35)$, such that TTC for $1.0^{*}(\bar{x}=1.26 \pm 0.34 \mathrm{~s})$ and $1.1^{*}(\bar{x}=1.22 \pm 0.3 \mathrm{~s})$ were significantly lower than $1.2^{*}(\bar{x}=1.31 \pm 0.34 s ; p=.03$ and $p=.005$ respectively $)$. TTC for $1.0^{*}$ and $1.1^{*}$ were not significantly different from each other $(p=1.0)$. Further, there was

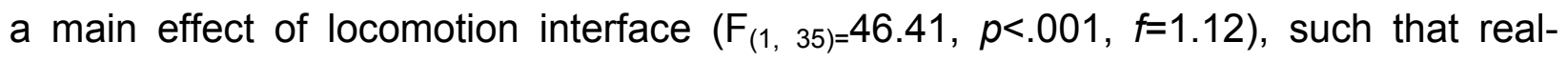


walking TTC $(\bar{x}=1.15 \pm 0.32 s)$ was significantly lower than joystick $(\bar{x}=1.35 \pm 0.33$ s; $p<.001)$. However, there was no interaction effect $(p=.188)$ (Figure 3$)$.

The results to determine if there was a block effect revealed that block order (1 through 4, regardless of locomotion interface) did not have an effect on the $50 \%$ Switch Point $\left(F_{(1.9,31)}=0.23, p=.79\right)$. There was also no block order effect on TTC values during the 1.0* condition $\left(F_{(3,32)}=1.17, p=.32\right)$, the $1.1^{*}$ condition $\left(F_{(2.29,32)}=1.16, p=.32\right)$, or the $1.2^{*}$ condition $\left(\mathrm{F}_{(2.4,32)}=2.4, p=.09\right)$, suggesting repeated exposures did not impact the data.

\subsection{Discussion}

The objective of this study was to determine whether aperture crossing behaviour was consistent between two different locomotion methods: real-walking and joystickcontrolled. Real-walking locomotion requires integrated perceptual information from the somatosensory, visual, and vestibular systems, whereas joystick-controlled locomotion creates incongruence between visual and non-visual information. During the task, participants made a decision regarding passability while approaching a closing aperture at a constant speed, and followed through with their decision (whether a pass through or a stop). This required participants to make decisions based on their own capabilities, and not only the features of the objects in the virtual environment. The task was designed to determine if it was possible to complete a closing gap aperture crossing task accurately using visual information in absence of congruent vestibular and somatosensory information, or whether these sensory inputs were needed for triangulation of visual information. Since vision is significantly up-weighted during VR 
RUNNING HEADER: SENSORY CONFLICT APERTURE CROSSING

locomotion, it was hypothesized that the $50 \%$ Switch Point and Time to Contact would be similar between real-walking and joystick-controlled locomotion. Gibson (1979) suggested that the optic array, which is directly available to a retina from the environment, is all that is necessary to inform appropriate actions. If the task produced similar behaviours between joystick-controlled locomotion and real-walking, then it suggests the optic array is upheld. Contrary to our hypothesis, a number of significant differences were observed between behavior using the two locomotion interfaces, therefore this suggests that non-visual information informs aperture crossing behaviours.

\section{$50 \%$ Switch Point}

Previous foundational VR research has concluded that real-walking while wearing an HMD is the preferred locomotion interface in terms of presence, ease of use, and naturalness (Boletsis, 2017). Further, physically interacting with the virtual environment triggers the most intuitive user responses (Boletsis, 2017), and therefore the $50 \%$ Switch Point of $0.8^{*}$ walking-speed (Figure 2) as determined in the present study likely reflects an individual's most natural response. Conversely, a 50\% switch point of $0.86^{*}$ walking-speed (Figure 2 ) as observed in the joystick-controlled locomotion, would indicate that individuals pass through apertures (gaps) closing slightly faster than real-walking. However, a lack of congruent somatosensory and vestibular information may not fully explain the differences found in this study without considering the role of vision. One study that presented a novel Locomotor Sensory Organization Test discovered that when somatosensory and/or vestibular input is manipulated during locomotion, the presence of accurate vision greatly reduces the potential for gait 
instability (Chien et al., 2014). Therefore, the behavioural differences observed in the current study may arise from changes to how visual information is utilized when there is sensory conflict, rather than a direct effect of vision being incongruent with somatosensory and vestibular perceptual inputs. Considering the role of vision on performance during this task, information about body and self-motion from the visual system is given higher priority over information from other sensory systems during locomotion (Chien et al., 2014; Patla, 1997). Further, when somatosensory or vestibular sensory inputs are incongruent with visual input, the nervous system will re-weight that information and upweight visual input to maintain balance, (Woollacott, Shumway-Cook, \& Nashner, 2005), a conclusion that has also been observed in virtual environments (Lubetzky, Harel, Darmanin, \& Perlin, 2016). In the present study, the upweighting of visual information may be problematic. When estimating travelled distance in a virtual environment using accurate sensory input from all senses, individuals provide accurate estimations, but underestimate distance travelled when making decisions purely based on visual information (Campos, Butler, \& Bülthoff, 2014; Caramenti et al., 2019). It is possible that when using joystick-controlled locomotion, the individuals in the current study underestimated the distance between themselves and the doors, which resulted in perceived successful passage through faster door closing rates than during realwalking. As a result, the absence of complementary proprioceptive and vestibular input creates sensory conflict, and the up-weighted visual system is unable to perform completely normally without congruence of non-visual information. Another plausible explanation is that the two different types of locomotion interfaces resulted in differing vection perceptions (i.e., the experience or illusion of self-motion) (Palmisano, Allison, 
Schira, \& Barry, 2015). In turn, this may have affected participants judgement of selfmotion during the trials and resulted in a differing switch point between the interfaces.

It is advantageous to compare these results to real life examples, however the majority of past research studying behaviour during aperture crossing used a critical point to determine performance. Critical point is defined as the point where the properties of the obstacle cause an individual to change their actions (Warren \& Whang, 1987). Critical point divides performance into possible and impossible, however it does not necessarily portray what people can do consistently. Modelling affordances as a probabilistic function (i.e., $50 \%$ Switch Point generated from a polynomial) addresses the variable nature of performance. Instead of dividing performance into success and failure, a continuous function represents the probability of success at each unit of the environment (Franchak \& Adolph, 2014). The 50\% Switch Points calculated in the present study represent a threshold where behaviour changes, and the proportion of door passage is plotted at each door closure condition (Figure 2). A simple way to determine similarity in aperture crossing behaviour between VR and real life is to compare a $50 \%$ switch point to the equivalent critical point. For example, critical point, or door width compared to shoulder width for a $50 \%$ switch point of $0.8^{*}$ (i.e., as found for real-walking), is equal to the aperture width at time of crossing divided by average shoulder width. The calculation to convert $50 \%$ switch point of $0.8^{*}$ walking speed to critical point $(\mathrm{CP})$ is given by:

$$
C P=\left(\frac{\left(W_{\text {door }}-\left(V_{\text {door }} \times T_{\text {door }}\right)\right)}{\overline{S W}}\right) * 100
$$

Where $T_{\text {door }}$ is the time (sec) in which the participant reaches the threshold of the doors, determined by dividing the $4 \mathrm{~m}$ approach distance by the average walking speed of the 
participants $(1.4 \mathrm{~m} / \mathrm{s}) . V_{\text {door }}$ is the rate at which the gap of the doors closed, determined by multiplying average walking speed $(1.4 \mathrm{~m} / \mathrm{s})$ by the $50 \%$ Switch Point $\left(0.8^{*}\right.$ or $0.86^{*}$ walking speed). $W_{\text {door }}$ is the starting width of the door aperture $(4 \mathrm{~m})$. Finally, average shoulder width $(\overline{S W})$ in this study was calculated to be $44.7 \mathrm{~cm}$. The resulting CP for real-walking $\left(0.8^{*}\right.$ switch point) was $1.7 x$ shoulder width (i.e., $76 \mathrm{~cm}$ aperture), and the CP for joystick-controlled locomotion $\left(0.86^{*}\right.$ switch point) was $1.16 \mathrm{x}$ shoulder width (i.e., $52 \mathrm{~cm}$ aperture). Based on the above calculation, the aperture closing speed that represented a critical point of $1.0 x$ shoulder width was determined to be $0.878 x$ walking speed. This value can be considered the threshold between passable and impassable without a shoulder rotation, or successful and unsuccessful aperturecrossing decisions. Since the switch point for both real-walking and joystick-controlled locomotion was lower than $0.878 \mathrm{x}$ and no collisions were recorded, it can be concluded that this task was performed successfully using both locomotion interfaces.

Most research concerning crossing of stationary apertures in real laboratory spaces find the critical point of young adults to be $1.4 x$ shoulder width (Hackney \& Cinelli, 2011; Warren \& Whang, 1987; Wilmut \& Barnett, 2010). However, when considering moving obstacles, there is a paucity of comparable studies. Some researchers observed aperture crossing behaviours in response to oscillating doors, but looked at other behavioural measures (Montagne et al., 2003), or found that individuals almost always chose to pass through when the oscillating aperture was opening, not closing (Cinelli \& Patla, 2008). Others have determined aperture crossing behaviours with two objects converging on a $45^{\circ}$ angle toward the participant, unlike the current study (Watson et al., 2011). Thus, the current paradigm presents a new metric to 
RUNNING HEADER: SENSORY CONFLICT APERTURE CROSSING

understand aperture crossing behaviour in VR, particularly for crossing closing gaps. Further, this knowledge is specific to which sensory systems are contributing to performance on the task, whether all sensory inputs are available (i.e., real-walking), or when only visual input is available (i.e., joystick-controlled). Based on the calculated critical point of $1.7 \times \mathrm{SW}$ during real-walking locomotion, when crossing a closing aperture in VR, individuals will pass through apertures that are at least $76 \mathrm{~cm}$ wide on average. Comparatively, when using joystick-controlled locomotion (1.16x SW), individuals will pass through apertures at least $52 \mathrm{~cm}$ wide, meaning they cross closing apertures with less medial-lateral clearance between the doors (i.e., distance between themselves and either door). Accordingly, we recommend that future VR paradigms which aim to study aperture crossing behaviour can effectively use a joystick-controlled locomotion interface and acknowledge the differences in crossing behaviour as a lack of congruence between visual and non-visual information when analyzing results. Despite the perceptual differences identified in this study, the ability to use joystick-controlled locomotion to study behaviour may be favorable in some situation. For instance, it enables researchers to study the visual control of locomotion and how collision avoidance is affected when physically completing the task may be impossible (i.e., room constraints).

\section{Time to Contact (TTC)}

TTC was only calculated for the conditions in which participants stopped $100 \%$ of the time. The TTC value represents how much time remained during the participants' approach before they would have reached the doors' threshold. Inversely, it is a measure of how much time was required to make a decision of passability based on 
visual information of the closing gap, and to stop the approach (where a smaller value indicates longer decision-making time). Of the three door closing conditions chosen for this analysis $\left(1.0^{*}, 1.1^{*}, 1.2^{*}\right)$, the $1.2^{*}$ condition was considered to be the easiest for passability decision-making, as it resulted in significantly larger TTC value (i.e., earlier decision) (Figure 3). The difference in TTC values between the door closing conditions is most likely the result of participants using optical variables (i.e., rate of change in beta angle, $\dot{\beta}$ ) to determine when a door was not passable and to initiation a stop (Fajen \& Warren, 2007).

A significant difference in TTC between locomotion types was determined in all three door closing conditions, such that participants consistently stopped closer to the aperture while using the real-walking interface compared to when using joystickcontrolled locomotion (Figure 3). This difference in TTC can be attributed to an inherent mechanical difference that may be present in the locomotion types (Brogan \& Johnson, 2003). During joystick-controlled locomotion, a decision to stop was performed by releasing the joystick trigger, and a stop occurred immediately. In the walking condition, once a decision to stop was made, gait termination was required, which required additional time to produce. The different mechanisms used to produce the action may explain the small but significant difference in TTC in the real-walking condition compared to the joystick-controlled condition. In order to control for a mechanical difference, acceleration and deceleration functions should be built into future joystickcontrolled paradigms to better simulate natural human locomotion and braking (Brogan \& Johnson, 2003). 
RUNNING HEADER: SENSORY CONFLICT APERTURE CROSSING

\section{Conclusion}

In conclusion, passability decision-making (i.e., $50 \%$ switch point) during a closing gap aperture crossing task in virtual reality is affected by locomotion interface. Despite the behaviour differences between locomotion interfaces, participants were equally successful on the task using both real-walking and joystick-controlled locomotion. Thus, future research can successfully use joystick-controlled locomotion to study behaviour in VR, and should take into account possible differences due to a lack of congruence between visual and non-visual information. 
RUNNING HEADER: SENSORY CONFLICT APERTURE CROSSING

\section{References}

Assländer, L., \& Peterka, R. J. (2016). Sensory reweighting dynamics following removal and addition of visual and proprioceptive cues. Journal of Neurophysiology, 116(2), 272-285. https://doi.org/10.1152/jn.01145.2015

Boletsis, C. (2017). The New Era of Virtual Reality Locomotion: A Systematic Literature Review of Techniques and a Proposed Typology. Multimodal Technologies and Interaction, 1(4), 24. https://doi.org/10.3390/mti1040024

Brogan, D. C., \& Johnson, N. L. (2003). Realistic human walking paths. Proceedings IEEE Workshop on Program Comprehension, 2003-Janua, 94-101. https://doi.org/10.1109/CASA.2003.1199309

Campos, J. L., Butler, J. S., \& Bülthoff, H. H. (2014). Contributions of visual and proprioceptive information to travelled distance estimation during changing sensory congruencies. Experimental Brain Research, 232(10), 3277-3289. https://doi.org/10.1007/s00221-014-4011-0

Caramenti, M., Lafortuna, C. L., Mugellini, E., Khaled, O. A., Bresciani, J. P., \& Dubois, A. (2019). Regular physical activity modulates perceived visual speed when running in treadmill-mediated virtual environments. PLOS ONE, 14(6), 1-14. https://doi.org/10.1371/journal.pone.0219017

Chien, J. H., Eikema, D. J. A., Mukherjee, M., \& Stergiou, N. (2014). Locomotor Sensory Organization Test: A Novel Paradigm for the Assessment of Sensory Contributions in Gait. Annals of Biomedical Engineering, 42(12), 2512-2523. https://doi.org/10.1007/s10439-014-1112-7

Cinelli, M. E., Patla, A. E., \& Allard, F. (2008). Strategies used to walk through a moving 
RUNNING HEADER: SENSORY CONFLICT APERTURE CROSSING

aperture. Gait and Posture, 27(4), 595-602.

https://doi.org/10.1016/j.gaitpost.2007.08.002

Cinelli, M. E., \& Patla, A. E. (2008). Task-specific modulations of locomotor action parameters based on on-line visual information during collision avoidance with moving objects. Human Movement Science, 27(3), 513-531.

https://doi.org/10.1016/j.humov.2007.10.001

Cirio, G., Olivier, A. H., Marchal, M., \& Pettré, J. (2013). Kinematic evaluation of virtual walking trajectories. IEEE Transactions on Visualization and Computer Graphics, 19(4), 671-680. https://doi.org/10.1109/TVCG.2013.34

Fajen, B. R. (2013). Guiding locomotion in complex, dynamic environments. Frontiers in Behavioral Neuroscience, 7(July), 1-15. https://doi.org/10.3389/fnbeh.2013.00085

Fajen, B. R., \& Matthis, J. S. (2011). Direct Perception of Action-Scaled Affordances: The Shrinking Gap Problem. Journal of Experimental Psychology: Human Perception and Performance, 37(5), 1442-1457. https://doi.org/10.1037/a0023510 Franchak, J., \& Adolph, K. (2014). Affordances as Probabilistic Functions: Implications for Development, Perception, and Decisions for Action. Ecological Psychology, 26(1-2), 109-124. https://doi.org/10.1080/10407413.2014.874923

Gibson, J. J. (2009). Reprinted from The British Journal of Psychology (1958), 49, 182194: Visually controlled locomotion and visual orientation in animals. British Journal of Psychology (London, England: 1953), 100(Pt 1A), 259-271. https://doi.org/10.1348/000712608X336077

Hackney, A. L., \& Cinelli, M. E. (2011). Action strategies of older adults walking through apertures. Gait and Posture, 33(4), 733-736. 
RUNNING HEADER: SENSORY CONFLICT APERTURE CROSSING

https://doi.org/10.1016/j.gaitpost.2011.02.019

Hackney, A. L., Cinelli, M. E., \& Frank, J. S. (2014). Is the critical point for aperture crossing adapted to the person-plus-object system? Journal of Motor Behavior, 46(5), 319-327. https://doi.org/10.1080/00222895.2014.913002

Karn, T., \& Cinelli, M. E. (2018). The effect of galvanic vestibular stimulation on path trajectory during a path integration task. Quarterly Journal of Experimental Psychology, 174702181879882. https://doi.org/10.1177/1747021818798824

Lee, D. N. (2012). Guiding movement by coupling taus. Closing the Gap: The Scientific Writings of David N. Lee, 7413(December), 139-174.

https://doi.org/10.4324/9780203936672

Lee, D. N., \& Kalmus, H. (1980). The Optic Flow Field : The Foundation of Vision [ and Discussion ]. Philosophical Transactions of the Royal Society of London, 290(1038), 169-179.

Lubetzky, A. V., Harel, D., Darmanin, H., \& Perlin, K. (2016). Assessment via the Oculus of Visual "Weighting" and "Reweighting" in Young Adults. Motor Control, 21(4), 468-482. https://doi.org/10.1123/mc.2016-0045

Montagne, G., Buekers, M., Camachon, C., De Rugy, A., \& Laurent, M. (2003). The learning of goal-directed locomotion: A perception-action perspective. Quarterly Journal of Experimental Psychology Section A: Human Experimental Psychology, 56 A(3), 551-567. https://doi.org/10.1080/02724980244000620

Montufar, J., Arango, J., Porter, M., \& Nakagawa, S. (2008). Pedestrians' Normal Walking Speed and Speed When Crossing a Street. Transportation Research Record: Journal of the Transportation Research Board, 2002(1), 90-97. 
RUNNING HEADER: SENSORY CONFLICT APERTURE CROSSING

https://doi.org/10.3141/2002-12

Palmisano, S., Allison, R. S., Schira, M. M., \& Barry, R. J. (2015). Future challenges for vection research: Definitions, functional significance, measures, and neural bases. Frontiers in Psychology, 6(FEB), 1-15. https://doi.org/10.3389/fpsyg.2015.00193

Patla, A. E. (1997). Understanding the roles of vision in the control of human locomotion.

Slater, M., Usoh, M., \& Steed, A. (1995). Taking steps: the influence of a walking technique on presence in virtual reality. ACM Transactions on Computer-Human Interaction, 2(3), 201-219. https://doi.org/10.1145/210079.210084

van Veen, H. A. H. C., Distler, H. K., Braun, S. J., \& Bülthoff, H. H. (1998). Navigating through a virtual city: Using virtual reality technology to study human action and perception. Future Generation Computer Systems, 14(3-4), 231-242.

https://doi.org/10.1016/s0167-739x(98)00027-2

Warren, W. H., \& Whang, S. (1987). Visual Guidance of Walking Through Apertures: Body-Scaled Information for Affordances. Journal of Experimental Psychology: Human Perception and Performance, 13(3), 371-383. https://doi.org/10.1037/00961523.13.3.371

Watson, G., Brault, S., Kulpa, R., Bideau, B., Butterfield, J., \& Craig, C. (2011). Judging the "passability" of dynamic gaps in a virtual rugby environment. Human Movement Science, 30(5), 942-956. https://doi.org/10.1016/j.humov.2010.08.004

Wilmut, K., \& Barnett, A. L. (2010). Locomotor adjustments when navigating through apertures. Human Movement Science, 29(2), 289-298. https://doi.org/10.1016/j.humov.2010.01.001 


\section{RUNNING HEADER: SENSORY CONFLICT APERTURE CROSSING}

Woollacott, M. H., Shumway-Cook, A., \& Nashner, L. M. (2005). Aging and Posture Control: Changes in Sensory Organization and Muscular Coordination. The International Journal of Aging and Human Development, 23(2), 97-114. https://doi.org/10.2190/vxn3-n3rt-54jb-x16x 
RUNNING HEADER: SENSORY CONFLICT APERTURE CROSSING

\section{Figure Captions}

Figure 1. Simulated VR environment showing an open aperture. Participants would begin standing on the home square, and pass through the doors to reach the goal square.

Figure 2. Average proportion of trials $( \pm S D)$ for Joystick-controlled locomotion and realwalking where participants stopped prior to passing through the closing aperture for each aperture condition (rate of closing/rate of approach) with a 3rd order polynomial; average $50 \%$ Switch Point for each locomotion interface identified.

Figure 3. Mean time to Contact (TTC) and standard deviation bars for $1.0^{*}, 1.1^{*}$, and $1.2^{*}$ walking speed conditions. * indicates significant difference at $p<.05$. 


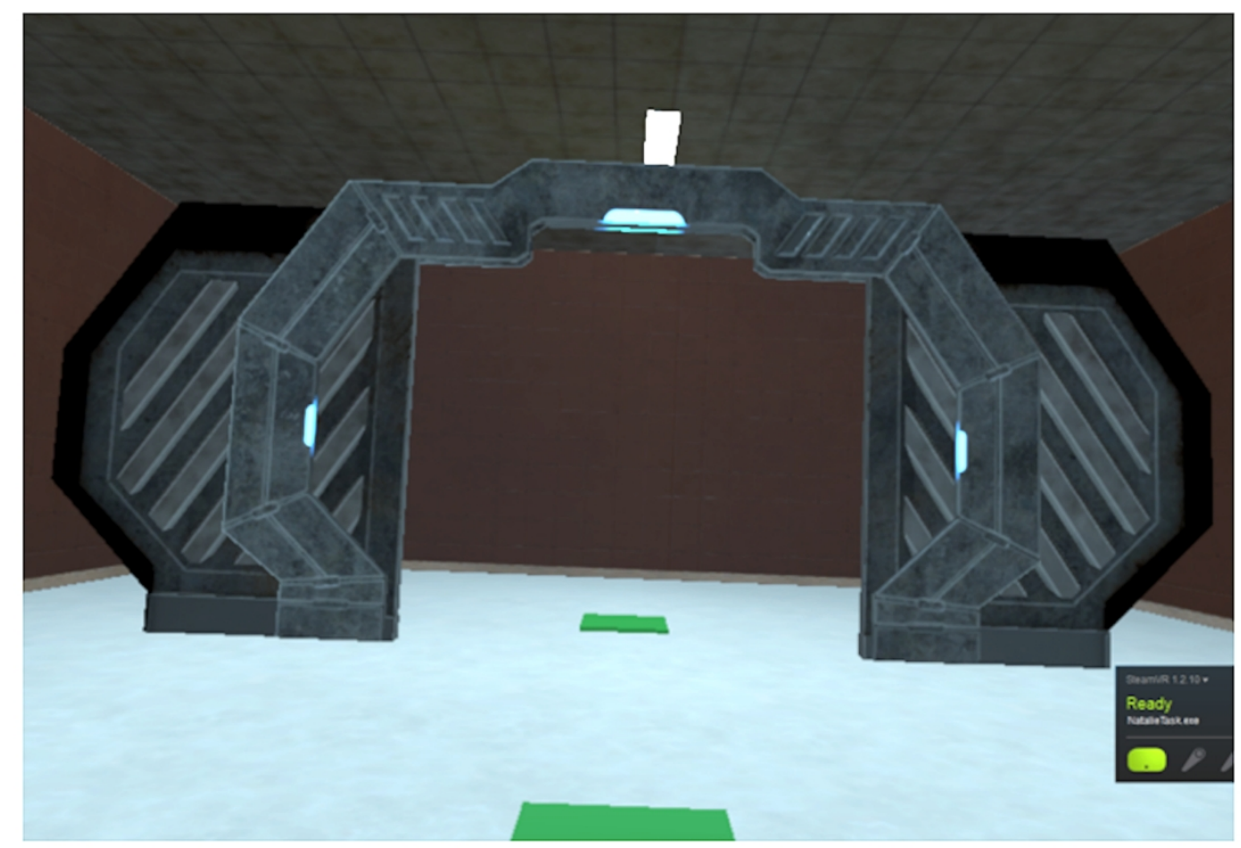

Figure 1

Figure 1. Simulated VR environment showing an open aperture. Participants would begin standing on the home square, and pass through the doors to reach the goal square.

\section{$101 \times 76 \mathrm{~mm}(1200 \times 1200 \mathrm{DPI})$}




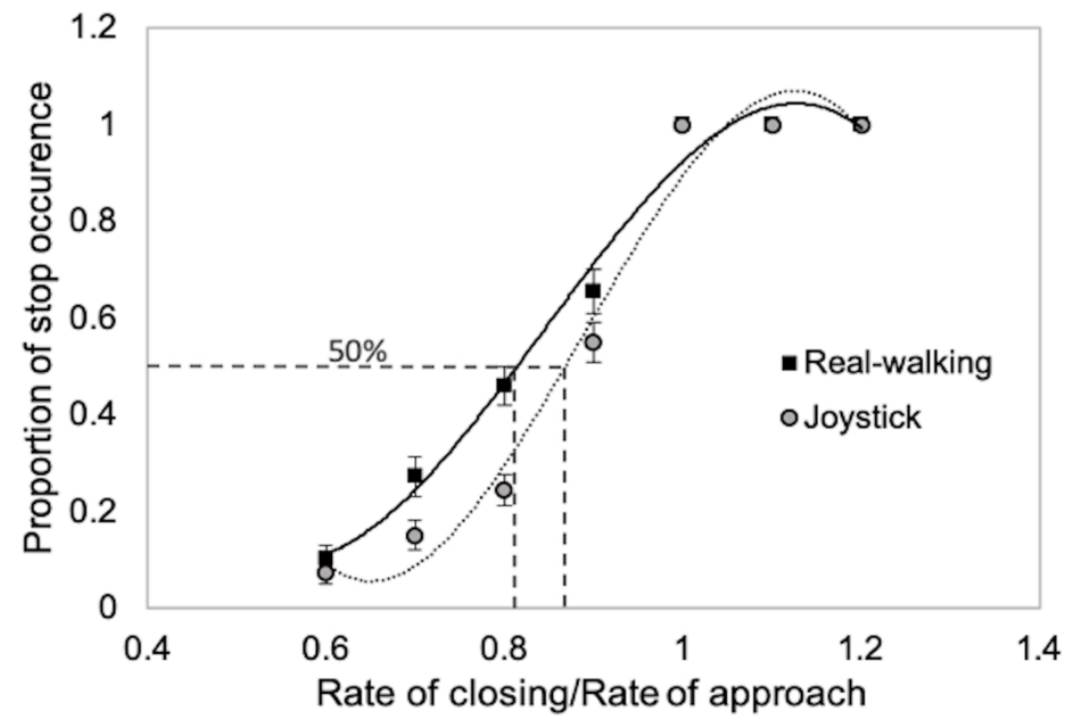

Figure 2

Figure 2. Average proportion of trials ( $\square$ SD) for Joystick-controlled locomotion and real-walking where participants stopped prior to passing through the closing aperture for each aperture condition (rate of closing/rate of approach) with a 3rd order polynomial; average 50\% Switch Point for each locomotion interface identified.

$152 \times 101 \mathrm{~mm}(600 \times 600 \mathrm{DPI})$ 


\begin{tabular}{|c|c|c|c|c|c|c|}
\hline \multirow{2}{*}{$\begin{array}{c}\text { Locomotion } \\
\text { Interface }\end{array}$} & \multicolumn{5}{|c|}{ Aperture Condition + Standard Deviation } \\
\cline { 2 - 7 } & $\mathbf{1 . 0}^{*}$ & SD & $\mathbf{1 . 1}^{\boldsymbol{*}}$ & SD & $\mathbf{1 . 2}^{\star}$ & SD \\
\hline Joystick & 1.3509 & 0.36169 & 1.2963 & 0.31824 & 1.3985 & 0.31273 \\
\hline Real-Walking & 1.0779 & 0.32299 & 1.1377 & 0.29683 & 1.218 & 0.37399 \\
\hline
\end{tabular}

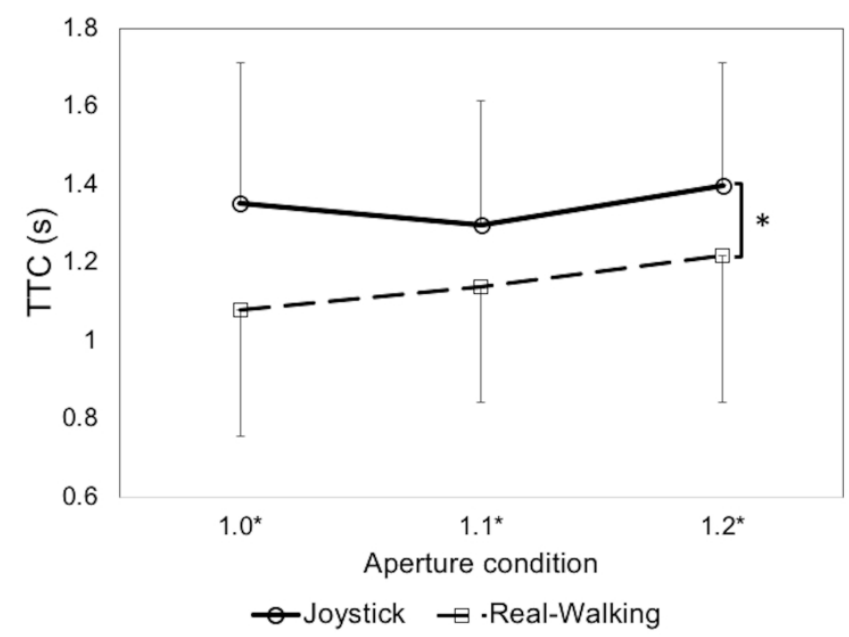

Figure 3

Figure 3. Mean time to Contact (TTC) and standard deviation bars for $1.0 *, 1.1 *$, and $1.2 *$ walking speed conditions. ${ }^{*}$ indicates significant difference at $\mathrm{p}<.05$.

$254 \times 190 \mathrm{~mm}(600 \times 600 \mathrm{DPI})$ 
Table 1. Participant characteristics presented as average \pm standard deviation.

\begin{tabular}{cc} 
Age (years) & $23.2 \pm 1.79$ \\
\hline Participants & $n=36$ \\
\hline Meight $(\mathrm{cm})$ & $172.16 \pm 10.35$ \\
\hline $\begin{array}{c}\text { Shoulder } \\
\text { width }(\mathrm{cm})\end{array}$ & $44.69 \pm 4.26$ \\
\hline $\begin{array}{c}\text { Walking } \\
\text { speed }(\mathrm{m} / \mathrm{s})\end{array}$ & $1.4 \pm 0.15$ \\
\hline
\end{tabular}

